\title{
MONTE CARLO SIMULATION MODEL OF ENERGY CONSUMPTION IN WHEATPRODUCTION SYSTEMS UNDER EGYPTIAN CONDITIONS
}

\section{G. M.Nasr ${ }^{1}$ - R. M. El-Kilani² - Anhar. M.Saeed ${ }^{3}$}

\begin{abstract}
The Monte Carlo simulation method was used in this study to examine uncertainty propagation in a modeled irrigated wheat production system in Egypt. After running the Monte Carlo Simulation for 10000 runs for each operation, the alternative with higher frequency in each operation was selected and the energy consumption was calculated. The result of the current study was new system in addition to the nineteen systems involved. The total energy consumption was 10205.09MJffed. This system consisted of Chisel plow 1st pass + Disc harrow + Steel leveler + Wooden Ridger + Mounted seed drill + Surface irrigation + Manual fertilizer Broadcasting + Manual operated knapsack sprayer + Combine. Among the nineteen systems involved in the study, the highest energy consumption system was system thirteen which consumed14650.8 MJ Fed. The system of minimum energy consumption within the 19 systems used in this study was S4 with 10109.174 MJ fed. This system consisted of Mould board Plow+ Disc harrow+ Wooden leveller+ Wooden ridger+ Mounted seed drill + Surface irrigation+ Manual fertilizer broadcasting + Manual operated knapsack Sprayer +Self-propelled mower+ Trailer + Stationary threshing machine. It can be concluded from the results of this study that Monte Carlo simulation model is capable of selecting the minimum energy consumption wheat production system.
\end{abstract}

Keywords: Monte Carlo simulation, agriculture, energy consumption, wheat

\footnotetext{
${ }^{1}$ Professor, Agric. Eng. Dept. Faculty of Agriculture - Cairo University

${ }^{2}$ Associate Professor, Soils and water Dept. Faculty of Agriculture - Cairo University

${ }^{3}$ Ph. D Student, Agric. Eng. Dept. Faculty of Agriculture - Cairo University
} 


\section{INTRODUCTION}

$\mathrm{E}$ nergy plays a major role in providing vital services, which are necessary for human survival and growth of civilization. Energy is a kind of strategic resource and is an important essential basis for economic increase and social development. In fact, per capita energy consumption is an index of growth of any nation in all forms (Asakerehet al., 2010)Energy use in agriculture has been increased in response to increasing population, limited supply of arable land, desire for higher standards of living (Kizilaslan, 2009) and modern farming has become very energy intensive. Nowadays, farmers use more energy to increase output, but due to inadequate knowledge and some mismanagement on using energy inputs, they lead to waste of energy resources and side negative effects. There is an urgent need to assess how energy resources are used, especially in developing countries. Energy consumption in agriculture is directly related to the development of technology in farming and the level of mechanization (Davoodi and Houshyar 2009). However, more intensive energy use has brought some important human health and environment problems so efficient use of inputs has become important in terms of sustainable agricultural production (Yilmazet al., 2005).

Agriculture is both a user and producer of energy and all agricultural operations require energy in one form or another: human power, animal power, fertilizer, fuels and electricity. Effective energy use in agriculture is one condition for sustainable agricultural production, since it provides financial savings, fossil resources preservation and air pollution reduction (Karimi et al., 2008). Finding a solution to reduce energy consumption per production unit seems to be essential to reach the sustainable development and to save the environment of future generation (Mohammadi et al., 2008).

Energy use in agriculture has paid very little attention (Pellizzi,1992). Energy modelling is an interesting subject among engineers and scientists who are concerned about energy production and consumption and environmental impacts (Tester, 2005and Al-Ghandoor et al., 2009).

Wheat is the most widely grown cereal crop in the world .Moreover; it has been considered the first strategic food crop. It is the main diet for the 
Egyptian population. Wheat is the main winter cereal crop and is widely distributed all over the country (Abd EI-Ghanyet al., 2012). According to FAO (2012) wheat is cultivated in Egypt on 1, 350, 000hectare with a production of $8,796,000$ tons with an average yield of 6.51 tons per hectare.

The term 'Monte Carlo' was first coined by Ulam and Von Neuman (Rubinstein, 1981) during their work on development of the atomic bomb during World War II (Bonate, 2001).

The name Monte Carlo comes from the gambling scene at the Monte Carlo Casino, Monaco for the simulation's random number generation feature. Monte Carlo simulation is a stochastic sampling method, where parameter values (e.g. inputs) are randomly generated from probability density function (PDFs) for multiple iterations. (PDFs) are often modeled on observed data, but can be developed from expert opinion (Winston 1991, Ayyub and Klir 2006).

Monte Carlo simulation is the term applied to stochastic simulations, either discrete, real-time, or some combination thereof, that incorporate random variability into the model.

Simulation affects our life every day through our interactions with the automobile, airline and entertainment industries, just to name a few. Monte Carlo simulation differs from traditional simulation in that the model parameters are treated as stochastic or random variables, rather than as fixed values (Bonate, 2001).

The performance and behavior of crop models is commonly made through comparison of simulated and observed variables. (Singh, et al.2013).In general terms, the Monte Carlo method of simulation can be used to describe any technique that approximates solutions to quantitative problems through statistical sampling "Probabilistic Simulation". Monte Carlo simulation can be considered as a methodical way of doing the socalled what-if analysis. (Raychaudhuri, 2008).

Two types of simulation are seen in research, deterministic and stochastic. Stochastic simulation looks at the long term effect of random variability in the model parameters on the outcome of a model. Deterministic simulation is a special case of stochastic simulation where variability is set equal to zero. As such, there is only one possible 
outcome for a set of inputs. Monte Carlo simulation differs from deterministic simulation in that the variability of the model parameters is included in the model and the long term impact of that variability is examined (Bonat, 2001).

A Monte Carlo simulation is a statistical simulation technique that provides approximate solutions to problems expressed mathematically. It utilizes a sequence of random numbers to perform this simulation. This technique can be used in different domains:

- Complex integral computations

- Economics specially in risk management

- Making decisions in specific complex problems (Gupta, 2011).

There are two types of problem to which we can apply Monte Carlo methods. The first type is that of purely stochastic problems where it seems natural that a solution may be found by making random number selection mimic the inherent randomness of the physical behavior. The second type is where we replace a deterministic problem, or one with no obvious random behavior, with a stochastic model whose average over many trials gives the same solution. Another important application of the Monte Carlo process, which is often related to the solution of a physical problem, is the evaluation of multi-dimensional integrals. In this application what is obtained is not only an estimate of the integral but also a standard deviation which is a measure of the uncertainty of the estimate. This is a characteristic of all Monte Carlo calculations; one always obtains an estimate and a deviation, and the reduction of the standard deviation, or variance, is an important aspect of the application of the method (Woolfson and Pert, 1999).

Monte Carlo simulation is a method for valuing derivatives that has achieved much interest during the last decades. It is a flexible method that has proved suitable to value complex financial instruments.

Monte Carlo Techniques (or Monte Carlo Methods, or in short Monte Carlo (MC)) are a class of techniques or algorithms that rely on the use of random sampling to finally acquire a solution to a given problem.

In principle, the Monte Carlo (MC) method can be considered as a very general mathematical tool for the solution of a great variety of problems. 
The applications of Monte Carlo methods can be divided into two major groups. One consists of direct simulations of systems that are already statistical in their nature; in such cases it is not even necessary to have well defined mathematical equations that describe the behavior of the system. The second group consists of Monte Carlo methods devised for the solution of well-defined mathematical equations. In such cases the methods are used to solve the equations that describe the problem of interes(Jacoboni and Lugli 1989).

In a Monte Carlo simulation we attempt to follow the 'time dependence' of a model for which change, or growth, does not proceed in some rigorously predefined fashion (e.g. according to Newton's equations of motion) but rather in a stochastic manner which depends on a sequence of random numbers which is generated during the simulation. With a second, different sequence of random numbers the simulation will not give identical results but will yield values which agree with those obtained from the first sequence to within some 'statistical error' (Landau and Binder, 2000).

Any Monte Carlo method relies on the generation of a sequence of random numbers with given distribution probabilities. Such a technique takes advantage of the fact that nowadays any computer generates sequences of random numbers evenly distributed between 0 and 1 at a sufficiently fast rate.

Simulation optimization has gained wide acceptance among researchers and many approaches have been developed. Simulation modeling has the capability of representing complex real world systems and the constraints in detail. Huseby et al. (2013) presented a simulation approach for establishing environmental contour lines for use in structural analysis of marine structures. Villada and Olaya (2013) used simulation approach to evaluate alternative policies for increasing the security of natural gas supply in Colombia. Korytkowski et al. (2013) developed evolutionary simulation-based heuristics to construct near-optimal solutions for dispatching rule allocation.

The Monte Carlo simulation method was used in this study to examine uncertainty propagation in a modeled irrigated wheat production system in Egypt. 


\section{MATERIALS AND METHODS}

Some of the data used in this study was obtained from literature cited. The other data obtained from a field measurements conducted in Egypt in Minya algamh during the season of 2011- 2012. Some of these measurements were fuel consumption, as well as the ground speed and width of equipment. A questionnaire was done to collect data concerning agricultural operations, power, and kind, age of tractors, self-propelled mower, size and age of equipment. Also the questionnaire included Irrigation system, irrigation frequency, irrigation duration (h) and fuel consumption $(1 / \mathrm{h})$, number of labors, number of animals, variety of seeds and seeding rate, type and amounts of fertilizers, and pesticide. Finally questionnaire included yield of wheat (tones /fed) for each system in this study.

The data covers the inputs, such as human energy consumption, animal energy consumption, and fuel consumption in different operations from seed -bed preparation up to harvesting and threshing operations which are used in the production of irrigated wheat under Egyptian conditions. In this study, energy consumption in wheat production was analysed based on direct energy sources and indirect energy sources. Direct energy including human energy consumption, animal energy consumption, and fuel energy consumption. While indirect energy included fertilizers, pesticides and seed. The total energy input $(\mathrm{E})$ is determined from the sum of the amount of input factors (Ai) multiplied by appropriate energy conversion coefficients for that factor (Ci).

$$
\mathrm{E}=\Sigma(\mathrm{Ai} \mathrm{Ci})
$$

Human energy was calculated from the following equations:

$$
\begin{gathered}
E_{h}=\frac{0.1 \times n}{1.36 \times \text { A.F.C }} \times 3.6 \\
E_{h}=\frac{0.264 \times n}{\text { A.F.C }}
\end{gathered}
$$

\section{Where}

$\mathbf{E}_{\mathbf{h}}$ : Human energy (MJ/ fed).

$\mathrm{n}$ : Number of workers required for operation. 
A. F. C: Actual field capacity of the gang of workers (fed/h).

1.36: Coefficient for transformation from $\mathrm{HP}$ to $\mathrm{kW}$.

0.1: Human power (HP)

3.6: Coefficient for transformation from $\mathrm{kW}$.h to MJ

Animal energy was calculated from the following equations

$$
\begin{gathered}
\mathrm{Ea}=\frac{0.5 \times n}{1.36 \times \text { A.F.C }} \times 3.6 \\
\mathrm{Ea}=\frac{1.323 \times n}{\text { A.F.C }}
\end{gathered}
$$

\section{Where}

Ea : Animal energy (MJ/ fed).

$\mathrm{n}$ : Number of farm animals used

A. F. C : Actual field capacity of the team of animals (fed/h).

1.36: Coefficient for transformation from $\mathrm{HP}$ to $\mathrm{kW}$.

0.5: Animal (Oxen) power (HP).

3.6: Coefficient for transformation from $\mathrm{kW}$. $\mathrm{h}$ to $\mathrm{MJ}$

Energy consumed in operating the machinery calculated from the following equations:

$$
\begin{gathered}
E_{m}=\text { F. C } \times \frac{1}{60 \times 60} \times \rho_{f} \times C . V . \times 427 \times \frac{1}{75} \times \frac{1}{1.36} \times \frac{1}{A . F . C} \cdots \\
E_{m}=\frac{F . C \times 9.884}{A . F . C} \times 3.6 \cdots \\
E_{m}=\frac{35.58 \times F . C}{A . F . C}-\cdots---(7)
\end{gathered}
$$

\section{Where}

Em: Energy consumed in operating the machinery (MJ/fed).

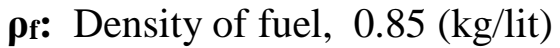

C.V. : Calorific value of fuel, $10000(\mathrm{kCal} . / \mathrm{kg})$

427: Constant (thermo -mechanical equivalent) (kg.m /kcal)

1.36: Coefficient for transformation from $\mathrm{HP}$ to $\mathrm{kW}$.

F.C: Fuel consumption $(1 / h)$.

A.F.C: Actual field capacity (fed/h).

3.6: Coefficient for transformation from $\mathrm{kW}$.h to $\mathrm{MJ}$ 
The data for energy equivalences were collected from various sources as indicated in table 1.

Table 1. Energy equivalents of inputs in wheat production.

\begin{tabular}{lcl}
\hline Input & Energy equivalent(MJ/kg) & \multicolumn{1}{c}{ Reference } \\
\hline Chemicals & & \\
$\quad$ Granular & 120.0 & Chaudhary et al. $(2006)$ \\
Topic & 271.38 & Mohammadi et al. (2008) \\
Pesticide & 280.44 & Mohammadi et al. $(2008)$ \\
$\begin{array}{l}\text { Organic fertilizer } \\
\quad \text { Manure }\end{array}$ & & \\
$\begin{array}{l}\text { Chemical fertilizers } \\
\text { Nitrogen }\end{array}$ & 0.3 & Verma(1987) \\
$\mathrm{P}_{2} \mathrm{O}_{5}$ & 66.14 & \\
Wheat seed & 12.44 & Shrestha (1998) \\
\hline
\end{tabular}

The operations for which energy inputs are estimated for on - farm production systems include, seedbed preparation, leveling, dividing, seeding, irrigation, fertilizing, weed control, harvesting, transporting and threshing as shown in Table 2 by using nineteen alternative systems for wheat production.

Table 2. The different nineteen systems of wheat production

\begin{tabular}{ll}
\hline System & \multicolumn{1}{c}{ Components } \\
\hline S1 & Chisel plow $\left(1^{1 \mathrm{st}}\right.$ pass \& $2^{\text {nd }}$ pass $)+$ wooden leveller wooden ridger+ \\
& broadcasting seed manually + surface irrigation+ broadcasting \\
& fertilizer manually+ manually operated knapsack sprayer+ \\
& sickle+ trailer+ stationary threshing machine (traditional).
\end{tabular}

S2 Chisel plow ( $1^{\text {st }}$ pass $\& 2^{\text {nd }}$ pass $)+$ wooden leveller wooden ridger+ mounted seed drill+ surface irrigation +broadcasting fertilizer manually+ manually operated knapsack sprayer+ self-propelled mower +trailer+ stationary threshing machine.

S3 Chisel plow ( $1^{\text {st }}$ pass $\& 2^{\text {nd }}$ pass $)+$ steel leveller wooden ridger+ mounted seed drill+ irrigation by sprinkler system +broadcasting fertilizer mechanically + hydraulic sprayer + self-propelled mower +trailer+ stationary threshing machine.

S4 Mouldboard plow+ disc harrow +wooden leveler+ wooden ridger+ mounted seed drill+ surface irrigation + broadcasting fertilizer manually+ manually operated knapsack sprayer+ selfpropelled mower +trailer+ stationary threshing machine. 
S5 Mouldboard plow+ disc harrow +steel leveler+ wooden ridger+ mounted seed drill+ irrigation by sprinkler system +broadcasting fertilizer mechanically +hydraulic sprayer+ self-propelled mower +trailer+ stationary threshing machine.

S6 Disc plow + disc harrow +wooden leveler+ wooden ridger+ mounted seed drill+ surface irrigation + broadcasting fertilizer manually + manually operated knapsack sprayer+ self-propelled mower +trailer+ stationary threshing machine.

S7 Disc plow+ disc harrow +steel leveler+ wooden ridger+ mounted seed drill + irrigation by sprinkler system +broadcasting fertilizer mechanically + hydraulic sprayer + self-propelled mower +trailer+ stationary threshing machine.

S8 Chisel plow $\left(1^{\text {st }}\right.$ pass $\& 2^{\text {nd }}$ pass $)+$ steel leveler+ wooden ridger+ mounted seed drill+ surface irrigation + broadcasting fertilizer manually+ manually operated knapsack sprayer +tractor mounted mower +trailer+ stationary threshing machine.

S9 Chisel plow $\left(1^{\text {st }}\right.$ pass $\& 2^{\text {nd }}$ pass $)+$ steel leveller wooden ridger+ mounted seed drill+ irrigation by sprinkler system +broadcasting fertilizer mechanically + hydraulic sprayer+ tractor mounted mower +trailer+ stationary threshing machine.

S10 Mould board plow+ disc harrow +wooden leveler+ wooden ridger+ mounted seed drill+ surface irrigation + broadcasting fertilizer manually+ manually operated knapsack sprayer+ tractor mounted mower +trailer+ stationary threshing machine.

S11 Mould board plow+ disc harrow +steel leveler+ wooden ridger+ mounted seed drill+ irrigation by sprinkler system +broadcasting fertilizer mechanically thydraulic sprayer+ tractor mounted mower +trailer+ stationary threshing machine.

S12 Disc plow+ disc harrow +wooden leveler+ wooden ridger+ mounted seed drill+ surface irrigation + broadcasting fertilizer manually+ manually operated knapsack sprayer+ tractor mounted mower +trailer+ stationary threshing machine.

S13 Disc plow + disc harrow +steel leveler + wooden ridger+ mounted 
seed drill+ irrigation by sprinkler system +broadcasting fertilizer mechanically + hydraulic sprayer+ tractor mounted mower + trailer+ stationary threshing machine.

S14 Mouldboard plow +disc harrow +wooden leveler+ wooden ridger+ mounted seed drill+ surface irrigation + broadcasting fertilizer manually+ manually operated knapsack sprayer+ combine.

S15 Mould board plow+ disc harrow +steel leveler+ wooden ridger+ mounted seed drill+ irrigation by sprinkler system +broadcasting fertilizer mechanically +hydraulic sprayer+ combine.

S16 Disc plow+ disc harrow +wooden leveler+ wooden ridger+ mounted seed drill+ surface irrigation + broadcasting fertilizer manually+ manually operated knapsack sprayer+ combine.

S17 Chisel plow ( $1^{\text {st }}$ pass $\& 2^{\text {nd }}$ pass $)+$ steel leveller wooden ridger+ mounted seed drill+ irrigation by sprinkler system +broadcasting fertilizer mechanically +hydraulic sprayer+ combine.

S18 Disc plow+ disc harrow +steel leveler + wooden ridger+ mounted seed drill + irrigation by sprinkler system +broadcasting fertilizer manually+ hydraulic sprayer+ combine.

S19 Chisel plow $\left(1^{\text {st }}\right.$ pass $\& 2^{\text {nd }}$ pass $)$ +wooden leveler+ wooden ridger+ mounted seed drill+ surface irrigation + broadcasting fertilizer mechanically + manually operated knapsack sprayer +combine

Based on energy consumption as optimum criterion in this research work, Monte Carlo simulation was used to find the most reliable system among different systems that are already used for the agricultural operations planting wheat in Egypt.

\section{Monte Carlo simulation}

Law and Kelton (2000) provided a 10 steps simulation model. Fig (1) demonstrates the flow chart that describes these steps.

Step 1 problem formulation is setting the objectives of the study and the specific issues to be considered. Resources available for such a 


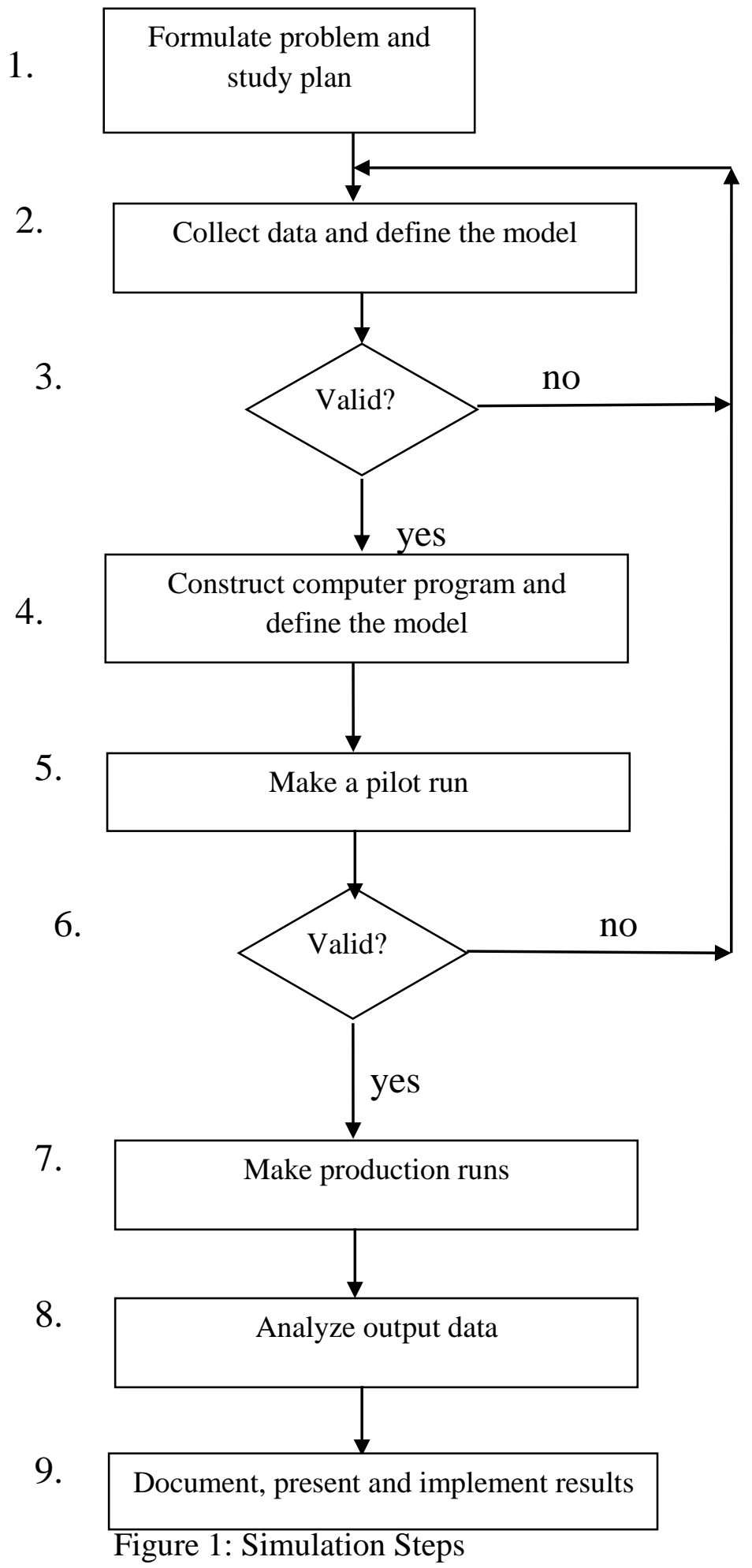


Study should also be considered, and expands on the importance of clarifying the issues to be considered; these include hardware design issues and operational issues. In addition, measures of performance have to be defined before starting the study. Step 2 in Fig. 1 is data collection. Data is collected if it exists based on the objectives of the study with the importance of data collection and stress the validation of such data which is step 3. After data is validated then step 4 is constructing a computer model, which is based on the conceptual model. After that a pilot run is done in step 5 the conceptual model is translated into a computerized model before starting step 6 and which is conducts the verification and the validation steps. It must be noted that most agree on the fact that the validation and verification process should be throughout the study. Steps 7 through 10 are design of experiments for defining the different alternatives for experimentation, production runs for providing performance data on systems designs of interest, output analysis which consists of statistical techniques for analyzing output from production runs, and implementation of models findings. We apply the simulation steps for our study in the following section.

\section{The application of Monte Carlo methodology}

This methodology is used to select the suitable method (way/alternative) in each operation of the possible agricultural systems.

The methodology consists of the following steps:

\section{Collecting data:}

1. Collect the data for the possible systems (S1 to S19)

2. In each system, the field operations machinery (seed bed preparation, Land leveling, Seeding, Irrigation, Fertilizing, Weed Control, and Harvesting) were determined with the possible operable alternatives.

3. For each alternative used in each operation, the consumed energy was determined. From the published relevant data, questionnaire or field measurement. As a typical examples of input data collection table 3 shows the operations of system1 (S1) and energy sources and consumption. 
Table 3.The operations of system1 (S1).

\begin{tabular}{|c|c|c|c|c|c|c|}
\hline \multirow{2}{*}{$\begin{array}{c}\text { S1 } \\
\text { Operation }\end{array}$} & \multicolumn{6}{|c|}{ Energy Consumption ( MJ / FED ) } \\
\hline & Machine & Human & Animal & Mechanical & Others & Total Energy \\
\hline \multirow{2}{*}{$\begin{array}{l}\text { Seed bed } \\
\text { preparation }\end{array}$} & $\begin{array}{l}\text { Chisel Plow } \\
1 \text { st pass }\end{array}$ & 0.30 & 0 & 155.01 & 0 & 155.31 \\
\hline & $\begin{array}{l}\text { Chisel Plow } \\
\text { 2st pass }\end{array}$ & 0.24 & 0 & 130.57 & 0 & 130.81 \\
\hline Levelling & $\begin{array}{l}\text { Wooden } \\
\text { Leveller }\end{array}$ & 1.29 & 6.44 & 0 & 0 & 7.73 \\
\hline Dividing & $\begin{array}{l}\text { Wooden } \\
\text { Ridger }\end{array}$ & 0.25 & 2.66 & 0 & 0 & 2.91 \\
\hline Seeding & $\begin{array}{l}\text { Manual broad } \\
\text { casting }\end{array}$ & 0.8 & 0 & 0 & 882 & 882.8 \\
\hline Irrigation & $\begin{array}{l}\text { Surface } \\
\text { irrigation }\end{array}$ & 1.26 & 0 & 523.852 & 0 & 525.112 \\
\hline Fertilizing & $\begin{array}{l}\text { Manual } \\
\text { broadcasting }\end{array}$ & 0.396 & 0.324 & 0 & 7997.1 & 7997.82 \\
\hline Weed Control & $\begin{array}{l}\text { Operated } \\
\text { knapsack } \\
\text { sprayer }\end{array}$ & 1.764 & 0 & 0 & 45 & 46.764 \\
\hline Harvesting & Sickle & 9.62 & 0 & 0 & 0 & 9.62 \\
\hline Transporting & Trailer & 0.64 & 0 & 153.64 & 0 & 154.28 \\
\hline Threshing & $\begin{array}{l}\text { Stationary } \\
\text { threshing } \\
\text { machine }\end{array}$ & 0.792 & 0 & 248.4 & 0 & 249.192 \\
\hline
\end{tabular}

After reviewing the 19 systems, they were classified according to how operations are performed as shown in table 4.

Creating Empirical Distributions:

1- For each alternative in each operation of the nineteen systems the frequency of using the implement and the related items were calculated to generate random number.

2- We compute the probability of each alternative and the cumulative probability. Table 5 shows the empirical distribution for seed bed preparation 1st passas an example.

Table 5. The empirical distribution for seed bed preparation 1st pass

\begin{tabular}{lcccc}
\hline $\begin{array}{l}\text { Operation } \\
\text { Implement }\end{array}$ & Chisel Plow 1st pas & $\begin{array}{c}\text { Seed bed preparation 1 } \\
\text { Mould Board plow }\end{array}$ & Disc plow & Total \\
\hline Frequency & 7 & 6 & 6 & 19 \\
Probability & 0.368421053 & 0.315789474 & 0.31578947 & 1 \\
Cummulative & 0.368421053 & 0.684210526 & 1 & \\
Range of & From zero & From 0.368 & From 0.684 & \\
R.N. & to 0.367 & to 0.683 & to 0.999 & \\
\hline
\end{tabular}


Table 4. Classification of the 19 systems according to how the operations are performed.

\begin{tabular}{|c|c|c|c|c|c|c|c|c|c|c|c|c|c|c|c|c|c|c|c|c|c|}
\hline & Equipment & S1 & S2 & S3 & S4 & S5 & S6 & S7 & S8 & S9 & S10 & S11 & S12 & S13 & S14 & S15 & S16 & S17 & S18 & S19 & Total \\
\hline \multirow{3}{*}{$\begin{array}{l}\text { Seed bed } \\
{\text { preparation } 1^{\text {st }}}^{\text {to }}\end{array}$} & Chisel Plow $1^{\text {st }}$ pas & $* *$ & $* *$ & $* *$ & & & & & $* *$ & $* *$ & & & & & & & & $* *$ & & $* *$ & 7 \\
\hline & Mould board plow & & & & $* *$ & $* *$ & & & & & $* *$ & $* *$ & & & $* *$ & $* *$ & & & & & 6 \\
\hline & Disc plow & & & & & & $* *$ & $* *$ & & & & & $* *$ & $* *$ & & & $* *$ & & $* *$ & & 6 \\
\hline \multirow{2}{*}{$\begin{array}{l}\text { seed bed } \\
{\text { preparation } 2^{\text {nd }}}\end{array}$} & Chisel Plow $2^{\text {nd }}$ pas & $* *$ & $* *$ & $* *$ & & & & & $* *$ & $* *$ & & & & & & & & $* *$ & & $* *$ & 7 \\
\hline & Disc Harrow & & & & $* *$ & $* *$ & $* *$ & $* *$ & & & $* *$ & $* *$ & $* *$ & $* *$ & $* *$ & $* *$ & $* *$ & & $* *$ & & 12 \\
\hline \multirow[t]{2}{*}{ Levelling } & Wooden Leveller & $* *$ & $* *$ & & $* *$ & & $* *$ & & & & $* *$ & & $* *$ & & $* *$ & & $* *$ & & & $* *$ & 9 \\
\hline & Steel Leveller & & & $* *$ & & $* *$ & & $* *$ & $* *$ & $* *$ & & $* *$ & & $* *$ & & $* *$ & & $* *$ & $* *$ & & 10 \\
\hline Dividing & Wooden Ridger & $* *$ & $* *$ & $* *$ & $* *$ & $* *$ & $* *$ & $* *$ & $* *$ & $* *$ & $* *$ & $* *$ & $* *$ & $* *$ & $* *$ & $* *$ & $* *$ & $* *$ & $* *$ & $* *$ & 19 \\
\hline \multirow[t]{2}{*}{ Seeding } & Manual broad casting & $* *$ & & & & & & & & & & & & & & & & & & & 1 \\
\hline & Mounted seed drill & & $* *$ & $* *$ & $* *$ & $* *$ & $* *$ & $* *$ & $* *$ & $* *$ & $* *$ & $* *$ & $* *$ & $* *$ & $* *$ & $* *$ & $* *$ & $* *$ & $* *$ & $* *$ & 18 \\
\hline \multirow[t]{2}{*}{ Irrigation } & Surface irrigation & $* *$ & $* *$ & & $* *$ & & $* *$ & & $* *$ & & $* *$ & & $* *$ & & $* *$ & & $* *$ & & & $* *$ & 10 \\
\hline & Sprinkler System & & & $* *$ & & $* *$ & & $* *$ & & $* *$ & & $* *$ & & $* *$ & & $* *$ & & $* *$ & $* *$ & & 9 \\
\hline \multirow[t]{2}{*}{ Fertilizing } & Manual broadcasting & $* *$ & $* *$ & & $* *$ & & $* *$ & & $* *$ & & $* *$ & & $* *$ & & $* *$ & & $* *$ & & $* *$ & & 10 \\
\hline & Mechanical Broad Casting & & & $* *$ & & $* *$ & & $* *$ & & $* *$ & & $* *$ & & $* *$ & & $* *$ & & $* *$ & & $* *$ & 9 \\
\hline \multirow[t]{2}{*}{ Weed Control } & Manually knapsack Sprayer & $* *$ & $* *$ & & $* *$ & & $* *$ & & $* *$ & & $* *$ & & $* *$ & & $* *$ & & $* *$ & & & $* *$ & 10 \\
\hline & Hydraulic Sprayer & & & $* *$ & & $* *$ & & $* *$ & & $* *$ & & $* *$ & & $* *$ & & $* *$ & & $* *$ & $* *$ & & 9 \\
\hline \multirow{4}{*}{ Harvesting } & Sickle & $* *$ & & & & & & & & & & & & & & & & & & & 1 \\
\hline & Self - Propelled Mower & & $* *$ & $* *$ & $* *$ & $* *$ & $* *$ & $* *$ & & & & & & & & & & & & & 6 \\
\hline & Tractor Mounted Mower & & & & & & & & $* *$ & $* *$ & $* *$ & $* *$ & $* *$ & $* *$ & & & & & & & 6 \\
\hline & combine & & & & & & & & & & & & & & $* *$ & $* *$ & $* *$ & $* *$ & $* *$ & $* *$ & 6 \\
\hline Transporting & Trailer & $* *$ & $* *$ & $* *$ & $* *$ & $* *$ & $* *$ & $* *$ & $* *$ & $* *$ & $* *$ & $* *$ & $* *$ & $* *$ & & & & & & & 13 \\
\hline Threshing & Stationary threshing machine & $* *$ & $* *$ & $* *$ & $* *$ & $* *$ & $* *$ & $* *$ & $* *$ & $* *$ & $* *$ & $* *$ & $* *$ & $* *$ & & & & & & & 13 \\
\hline
\end{tabular}




\section{Run the Monte Carlo Simulation:}

Table 6 shows the random number generated uniformly using function rand() and the alternative selected according to programming if statement=IF(G4<0.368;"chies11 st"; $\mathrm{IF}(\mathrm{G} 4<0.684$;"mouldboardplow"; $\mathrm{IF}($ $\mathrm{G} 4<=1$;"disc plow"))) where G4 the cell which contains the generated random number.

$=\mathrm{IF}(\mathrm{G} 4<0.368$;"chies11st"; $\mathrm{IF}(\mathrm{G} 4<0.684 ;$ "mould plow"; $\mathrm{IF}(\mathrm{G} 4<=1$; "disc plow")))

Where G4 has a random number $(=\mathrm{RAND}())$ uniformly distributed.

Table 6.The random number and the alternative

\begin{tabular}{lc}
\hline Random Number & Correspond implement \\
\hline 0.215410284 & chisel 1st \\
0.001367954 & chisel 1st \\
0.054564916 & chisel 1st \\
0.791211133 & disc plow \\
0.548898989 & mould plow \\
0.248796945 & chisel 1st \\
0.235178217 & chisel 1st \\
0.919362189 & disc plow \\
0.078832082 & chisel 1st \\
0.780873981 & disc plow \\
0.051430733 & chisel 1st \\
0.596983268 & mould plow \\
0.765539412 & disc plow \\
0.413287239 & mould plow \\
0.561557583 & mould plow \\
\hline
\end{tabular}

The previous procedure was repeated 10000 runs for each alternative implement and compute the new frequency and ratio. For example the result of land leveling after 10000 runs is shown in table 7.

Table 7.The result of land leveling after 10000 run

\begin{tabular}{llll}
\hline Equipment & Frequency & ratio & Total Energy \\
\hline Wooden leveler & 4652 & 0,465 & 7.73 \\
Steel leveler & 5348 & 0,535 & 114.186 \\
Total & 10000 & 1 & \\
\hline
\end{tabular}




\section{RESULT AND DISCUSSION}

Table 8 and Table 9 show the empirical distribution for Seed bed preparation (Primary tillage).It is clear that the chisel plow1st pass recorded higher frequency 4200, while disc plow recorded the lowest frequency2000.The same Monte Carlo technique used above for Seed bed preparation (primary tillage) was used for the following operations. seed bed preparation (secondary tillage ), leveling, dividing, seeding, irrigation, fertilizing, weed control, harvesting, transporting and threshing.

Table 8. The empirical distribution for Seed bed preparation (Primary tillage)

\begin{tabular}{lllll}
\hline The alternative & Frequency & $\begin{array}{c}\text { Probability of } \\
\text { occurance }\end{array}$ & $\begin{array}{c}\text { Cumulative } \\
\text { probability }\end{array}$ & $\begin{array}{c}\text { Rang of random } \\
\text { numbers }\end{array}$ \\
\hline Chisel plow 1st pass & 7 & 0.368 & 0.368 & From 000-367 \\
Mouldboard Plow & 6 & 0.316 & 0.684 & From 368-683 \\
Disc Plow & 6 & 0.316 & 1.000 & From 684-999 \\
Total & 19 & 1.000 & & \\
\hline
\end{tabular}

Table 9. The result of Seed bed preparation (Primary tillage) after 10000 simulation runs

\begin{tabular}{llll}
\hline Equipment & Frequency & Ratio & Total Energy $(\mathbf{M J} / \mathbf{F e d})$ \\
\hline Chisel 1 st $_{\text {pass }}$ & 4200 & 0.420 & 155.31 \\
Mouldboardplow & 3800 & 0.380 & 204.238 \\
Disc plow & 2000 & 0.200 & 232.127 \\
Total & 10000 & 1 & \\
\hline
\end{tabular}

\section{The output of Monte Carlo Simulation:}

After running the Monte Carlo Simulation for 10000 runs for each operation, the alternative with higher frequency in each operation was selected and the consumed energy was computed. The result of the current study suggested a new system in addition to the nineteen systems involved. The total energy consumption of this system was 10205.09 $\mathrm{MJ} / \mathrm{Fed}$. This system consisted of Chisel plow $1^{\text {st }}$ pass + Disc harrow+ Steel leveler + Wooden Ridger + Mounted seed drill + Surface irrigation + Manual fertilizer broadcasting + Knapsack sprayer + Combine as shown in table 10. 
Table10. The output of Monte Carlo Simulation after 10000 simulation runs

\begin{tabular}{|c|c|c|c|c|}
\hline Operation & Alternative & Frequency & Ratio & $\begin{array}{l}\text { Energy } \\
(\mathbf{M J} / \text { Fed })\end{array}$ \\
\hline $\begin{array}{l}\text { Seedbed } \\
\text { preparation } 1^{\text {st }}\end{array}$ & Chisel 1st pass (Three alternative) & 3685 & 0.369 & 155.31 \\
\hline $\begin{array}{l}\text { Seedbed } \\
\text { preparation } 2^{\text {nd }}\end{array}$ & Disc Harrow (Two alternative) & 6319 & 0.632 & 72.934 \\
\hline Land Leveling & Steel leveler (Two alternative) & 5348 & 0.535 & 114.186 \\
\hline Dividing & Wooden ridger (No alternative) & - & - & 2.91 \\
\hline Seeding & Mounted Seed drill (Two alternative) & 8945 & 0.895 & 810.56 \\
\hline Irrigation & Surface irrigation (Two alternative) & 5249 & 0.525 & 525.112 \\
\hline Fertilizing & $\begin{array}{l}\text { Manualfertilizer Broadcasting } \\
\text { (Two alternative) }\end{array}$ & 5303 & 0.530 & 7997.82 \\
\hline Weed Control & $\begin{array}{l}\text { Manual knapsack sprayer } \\
\text { (Two alternative) }\end{array}$ & 5276 & 0.528 & 46.764 \\
\hline Harvesting & Combine (Four alternative) & 3220 & 0.322 & 479.5 \\
\hline Total energy & & & & 10205.09 \\
\hline
\end{tabular}

The system of highest energy consumption within the 19 systems used in this study was $\mathrm{S}_{13}$ with $14650.8 \mathrm{MJ} /$ fed as shown in Table 11. This system consisted of disc plow, disc harrow, steel leveller, wooden ridger, seeding by mounted seed drill, irrigation by sprinkler system, mechanical fertilizer broadcasting, hydraulic sprayer for weed and pests control, tractor mounted mower, trailer and stationary threshing machine.

The maximum value of the highest energy consumption system within the 19 systems used in this study due to the application of disc plow, steel leveller, and irrigation by sprinkler system, mechanical fertilizer broadcasting, hydraulic sprayer and tractor mounted mower, and all of them (equipment or method) consumed high energy.

Table11. The highest energy consumption system within the 19 systems

\begin{tabular}{lcc}
\hline Operation & Equipment & $\begin{array}{c}\text { Maximum energy } \\
\text { consumption (MJ/Fed) }\end{array}$ \\
\hline $\begin{array}{l}\text { Seedbed preparation } \\
\text { (primary tillage ) }\end{array}$ & Disc plow & 232.127 \\
Seedbed preparation & & \\
(secondary tillage ) & Disc harrow & 72.934 \\
Leveling & Steel leveller & 114.186 \\
Dividing & Wooden Ridger & 2.91 \\
Seeding & Mounted seed drill & 810.56 \\
Irrigation & Irrigation by sprinkler system & 4249.68 \\
Fertilizing & Broadcastingfertilizer mechanically & 8072.66 \\
Weed Control & Hydraulic sprayer & 563.176 \\
Harvesting & Tractor mounted mower & 129.162 \\
Transporting & Trailer & 154.28 \\
Threshing & Stationary threshing & 249.192 \\
\hline Total energy & machine & $\mathbf{1 4 6 5 0 . 8}$ \\
\hline
\end{tabular}


The system of minimum energy consumption within the 19 systems used in this study was $\mathrm{S}_{4}$ with $10109.174 \mathrm{MJ} /$ fed as shown in Table 12. This system consisted of Mould board plow, disc harrow, wooden leveller, wooden ridger, irrigation by surface irrigation, seeding by mounted seed drill, Manual fertilizer broadcasting, and manual operated knapsack sprayer for weed and pests control, self-propelled mower, trailer and stationary threshing machine.

The minimum value of the minimum energy consumption system within the 19 systems used in this study due to the application of mould board Plow, disc harrow, wooden leveller, surface irrigation, mounted seed drill, manual fertilizer broadcasting, manual operated knapsack sprayer, self-propelled mower, trailer and stationary threshing machine, and all of them (equipment or method) consumed low energy.

Table12. The minimum energy consumption system within the 19 systems

Operation

Equipment

Mould board plow

Seed bed preparation

(primary tillage)

Seed bed preparation

(secondary tillage)

Levelling

Dividing

Seeding

Irrigation

Fertilizing

Weed control

Harvesting

Transporting

Threshing
Wooden leveller

Wooden ridger

Mounted seed drill

Surface irrigation

Manual broadcasting

Manual operated knapsack sprayer

Self - propelled mower

Trailer

Stationary threshing machine

Total
Maximum energy consumption MJ/fed

In this study a complete analysis of energy use in irrigated wheat production systems under Egyptian conditions is shown in Table 12, 13 and 14. 
Table12. Number of workers, animals, fuel consumption and consumed energy need for the execution of different operations.

\begin{tabular}{|c|c|c|c|c|c|c|c|c|}
\hline \multirow{2}{*}{$\begin{array}{l}\text { Equipment or } \\
\text { method }\end{array}$} & \multirow{2}{*}{ operation } & \multirow{2}{*}{$\begin{array}{c}\text { No. of } \\
\text { workers }\end{array}$} & \multirow{2}{*}{$\begin{array}{c}\text { No. of } \\
\text { animal } \\
\quad s\end{array}$} & \multirow{2}{*}{$\begin{array}{c}\text { Fuel } \\
\text { cons. } \\
(\mathbf{l} / \mathbf{h})\end{array}$} & \multirow{2}{*}{$\begin{array}{c}\text { Actual } \\
\text { field } \\
\text { capacit } \\
\text { y fed/h }\end{array}$} & \multicolumn{3}{|c|}{$\begin{array}{c}\text { Energy consumption } \\
(\mathrm{MJ} / \mathrm{Fed})\end{array}$} \\
\hline & & & & & & H. & A. & M. \\
\hline Chisel plow & $1^{\text {st }}$ pass & 1 & 0 & 3.79 & 0.87 & 0.30 & 0.00 & 155.01 \\
\hline Chisel plow & $2^{\text {nd }}$ pass & 1 & 0 & 4.00 & 1.09 & 0.24 & 0.00 & 130.57 \\
\hline Mouldboard plow & $1^{\text {st }}$ pass & 1 & 0 & 4.47 & 0.78 & 0.33 & 0.00 & 203.90 \\
\hline Disc plow & $1^{\text {st }}$ pass & 1 & 0 & 4.69 & 0.72 & 0.36 & 0.00 & 231.76 \\
\hline Disc harrow & $\begin{array}{l}\text { Secondary } \\
\text { tillage }\end{array}$ & 1 & 0 & 3.60 & 1.76 & 0.15 & 0.00 & 72.790 \\
\hline Wooden leveller & Levelling & 2 & 2 & 0 & 0.41 & 1.29 & 6.44 & 0.000 \\
\hline Steel leveller & levelling & 1 & 0 & 3.78 & 1.18 & 0.22 & 0.00 & 113.97 \\
\hline Wooden ridger & Dividing & 1 & 2 & 0 & 1 & 0.26 & 2.66 & 0.000 \\
\hline Manual broadcasting & Seeding & 2 & 0 & 0 & 0.67 & 0.8 & 0.00 & 0.000 \\
\hline Mounted seed- drill & Seeding & 1 & 0 & 3.73 & 1.76 & 0.15 & 0.00 & 75.420 \\
\hline Surface irrigation & Irrigation & 2 & 0 & 22.26 & 0.42 & 1.26 & 0 & 523.852 \\
\hline sprinkler system & Irrigation & 2 & 0 & 0 & 0.05 & 10.58 & 0 & $4239.10^{*}$ \\
\hline Manual broadcasting & Fertilizing & 2 & 1 & 0 & 1.33 & 0.39 & 0.324 & 0.000 \\
\hline Mechanical broadcasting & Fertilizing & 1 & 0 & 3.73 & 1.76 & 0.15 & 0.00 & 75.420 \\
\hline Manual knapsack sprayer & $\begin{array}{l}\text { Weed and pest } \\
\text { control }\end{array}$ & 1 & 0 & 0 & 0.150 & 1.76 & 0.00 & 0.000 \\
\hline Hydraulic sprayer & $\begin{array}{l}\text { Weed and pest } \\
\text { control }\end{array}$ & 2 & 0 & 6.320 & 0.435 & 1.216 & 0.00 & 516.96 \\
\hline Sickle & Harvesting & 4 & 0 & 0 & 0.11 & 9.62 & 0.00 & 0.000 \\
\hline Self - propelled mower & Harvesting & 2 & 0 & 0.62 & 0.60 & 0.882 & 0.00 & 36.75 \\
\hline Tractor - mounted mower & Harvesting & 2 & 0 & 3.84 & 1.060 & 0.49 & 0.00 & 128.91 \\
\hline Combine & Harvesting & 2 & 0 & 14.27 & 1.060 & 0.49 & 0.00 & 479.016 \\
\hline Trailer & Transporting & 2 & 0 & 2.85 & 0.82 & 0.64 & 0.00 & 153.64 \\
\hline $\begin{array}{l}\text { Stationary threshing } \\
\text { machine }\end{array}$ & Threshing & 2 & 0 & 3.70 & 0.66 & 0.792 & 0.00 & 248.4 \\
\hline
\end{tabular}

Where: H:Human,A: Animal, M: Mechanical,: Electricity*

Table 13. Amounts of inputs and energy inputs for wheat production

\begin{tabular}{lcc}
\hline Inputs & Quantity (kg/fed) & $\begin{array}{c}\text { Total energy equivalent } \\
(\mathbf{M J} / \text { fed) }\end{array}$ \\
\hline $\begin{array}{l}\text { Input } \\
\text { Chemical (kg) }\end{array} \quad$ & \\
$\quad$ Granular & & 0.96 \\
$\quad$ Topic & 0.0080 & 38 \\
$\quad$ Pesticide & 0.1400 & 5.94 \\
Organic fertilizer & 0.0212 & 2850 \\
$\quad$ Manure & & \\
Chemical fertilizer & 9500 & 4960.5 \\
Nitrogen (N) & & 186.6 \\
Superphosphate $\left(\mathrm{P}_{2} \mathrm{O}_{5}\right)$ & 75 & 882 \\
$\quad$ Wheat seed & 15 & 735 \\
Manual broadcasting & & \\
Mounted seed-drill & 60 & \\
\hline
\end{tabular}


Table 14. Analysis of energy use in irrigated wheat production systems under Egyptian conditions.

\begin{tabular}{cccccccc}
\hline Item & Human & Animal & Mechanical & Fertilizer & Seed & Chemical & Total energy input \\
\hline S1 & 17.34 & 9.424 & 1211.476 & 7997.1 & 882 & 45 & 10162.34 \\
S2 & 7.947 & 9.424 & 1323.646 & 7997.1 & 735 & 45 & 10118.117 \\
S3 & 15.392 & 2.66 & 5745.245 & 7997.1 & 735 & 45 & 14540.406 \\
S4 & 7.9 & 9.424 & 1314.75 & 7997.1 & 735 & 45 & 10109.174 \\
S5 & 15.346 & 2.66 & 5736.358 & 7997.1 & 735 & 45 & 14531.464 \\
S6 & 7.929 & 9.424 & 1342.61 & 7997.1 & 735 & 45 & 10137.063 \\
S7 & 15.375 & 2.66 & 5764.218 & 7997.1 & 735 & 45 & 14559.353 \\
S8 & 7.316 & 9.424 & 1415.806 & 7997.1 & 735 & 45 & 10209.646 \\
S9 & 14.762 & 2.66 & 5837.408 & 7997.1 & 735 & 45 & 14631.93 \\
S10 & 7.27 & 9.424 & 1406.91 & 7997.1 & 735 & 45 & 10200.704 \\
S11 & 14.716 & 2.66 & 5828.518 & 7997.1 & 735 & 45 & 14622.994 \\
S12 & 7.299 & 9.424 & 1434.77 & 7997.1 & 735 & 45 & 10228.593 \\
S13 & 14.745 & 2.66 & 5856.378 & 7997.1 & 735 & 45 & 14650.883 \\
S14 & 6.076 & 9.424 & 1354.97 & 7997.1 & 735 & 45 & 10147.57 \\
S15 & 13.522 & 2.66 & 5776.578 & 7997.1 & 735 & 45 & 14569.86 \\
S16 & 6.105 & 9.424 & 1382.83 & 7997.1 & 735 & 45 & 10175.459 \\
S17 & 13.568 & 2.66 & 5785.474 & 7997.1 & 735 & 45 & 14578.802 \\
S18 & 13.803 & 2.984 & 5729.018 & 7997.1 & 735 & 45 & 14522.905 \\
S19 & 5.87 & 9.1 & 1439.286 & 7997.1 & 735 & 45 & 10231.356 \\
\hline
\end{tabular}

\section{CONCLUSION}

After running the Monte Carlo Simulation for 10000 runs for each operation, we select the alternative with higher frequency in each operation and compute the energy consumed. The results of the current study introduced a new system in addition to the nineteen systems involved. The total energy consumption of this new system was $10205.09 \mathrm{MJ} / \mathrm{Fed}$. This system consisted of Chisel plow 1st pass+ Disc harrow+ Steel leveler + Wooden Ridger + Mounted seed drill + Surface irrigation + Manual fertilizer Broadcasting + Knapsack sprayer + Combine.

The highest energy consumption system within the nineteen systems used in this study was $\mathrm{S}_{13}$ with $14650.8 \mathrm{MJ} /$ Fed. This system consisted of disc plow, disc harrow, steel leveller, wooden ridger, irrigation by sprinkler system, mechanical fertilizer broadcasting, hydraulic sprayer for weed and pests control and tractor mounted mower, trailer and stationary threshing machine. The system of minimum energy consumption within the 19 systems used in this study was $\mathrm{S}_{4}$ with $10109.174 \mathrm{MJ} / \mathrm{fed}$. This system consisted of Mould board Plow + Disc harrow+ Wooden leveller + Wooden ridger+ Surface irrigation+ Mounted seed drill + Manual fertilizer 
broadcasting+ Manual operated knapsack sprayer + Self-propelled mower+ Trailer + Stationary threshing machine.

The results of this study proved the ability of Monte Carlo simulation model to predict energy consumption in wheat production systems.

\section{REFERENCE}

Abd EI-Ghany, H.M.; Abd EI-Salam, M.S.; Hozyen, M. and Afifi , M.H.M.(2012). Effect of deficit irrigation on some growth stages of wheat. Journal of applied sciences research, 8(5):27762784.ISSN 1819-544X.

Al-Ghandoor, A.; Jaber J.O.; Al-Hinti I. and Mansour I.M. (2009). Residential past and future energy consumption: Potential savings and environmental impact. Renewable and Sustainable Energy Reviews. 13(6-7):1262-74.

Asakereh, A.;Shiekhdavoodi, M.J. and Akram, A.(2010).Investigation of Energy Consumption in Agriculture Sector of Iran and their Effect on Air Pollution and Social Cost. Research Journal of Applied Sciences Engineering and Technology 2(5): 401-406, 2010 ISSN: 2040-7467.

Ayyub, B. and Klir, G. (2006). Uncertainty Modeling and Analysis in Engineering and the Sciences. Taylor \& Francis Group, Boca Raton, FL. 378p.

Bonate, P.L. (2001). A Brief Introduction to Monte Carlo Simulation. ClinPharmacokinet, 40 (1): 15-22.

Chaudhary, V.P.;Gangwar B, Pandey, D.K. (2006). Auditing of energy use and output of different cropping systems in India. Agricultural Engineering international the CIGR journal. 5: 1-13.

Davoodi, M.J. and Houshyar, E. ( 2009). Energy Consumption of Canola and Sunflower Production in Iran. American-Eurasian J. Agric. \& Environ. Sci., 6 (4): 381-384.

Embaby, A. T. (1985).A comparison of the different mechanization systems for cereal crop production. M.Sc. thesis, Agric. Eng. Dept. Faculty of agriculture. Cairo University. 
FAO(2012). FAO Stat, http://faostat.fao.org/default.aspx

Gupta, A. (2011). Monte Carlo simulations modeling and applications. Indian institute of technology, Kanpur, Indian.

Huseby, A. B.; Vanem, E. and Natvig, B. (2013). A new approach to environmental contours for ocean engineering applications based on direct Monte Carlo simulations, Ocean Engineering, 60, 124135 .

Jacoboni, C. and Lugli, P. (1989). The Monte Carlo Method for Semiconductor Device Simulation, Springer-VerJag/Wien, 1989.

Karimi, M.; Rajabi, P. A.; Tabatabaeefar, A. and Borghei, A. (2008). Energy Analysis of Sugarcane Production in Plant Orchards A Case Study in DebelKhazai Agro-industry in Iran. AmericanEurasian J. Agric. Environ. Sci., 4(2): 165-171.

Kizilaslan, H. (2009). Input-output energy analysis of cherries production in Tokat Province of Turkey. Applied Energy. 86: 1354-1358.

Korytkowski, P.; Wisniewski, T.;Rymaszewski, S., (2013). An evolutionary simulation-based optimization approach for dispatching scheduling, Simulation Modeling Practice and Theory, $35,69-85$

Landau, D. P. and Binder, K. A. (2000).Guide to Monte Carlo Simulations in Statistical Physics, Second Edition, Cambridge university press.

Law, A.M. and Kelton, W.D. (2000). Simulation Modeling and Analysis, Third ed., McGraw-Hill, New York.

Mohammadi, A.; Tabatabaeefar, A.; Shahin,S.;Rafiee, S. and Keyhani, A. (2008). Energy use and economic analysis of potato production in Iran a case study: Ardabil province. Energy Conversion Manage. 49:3566-3570.

Pellizzi, G. (1992). Use of energy and labour in Italian agriculture. Journal of Agricultural Engineering Research. 52:111-9. 
Raychaudhuri, S.(2008).Introduction to Monte Carlo Simulation. Proceedings of the 2008 Winter Simulation Conference.S. J. Mason, R. R. Hill, L. Mönch, O. Rose, T. Jefferson, J. W. Fowler eds. Oracle Crystal Ball Global Business Unit 390 Interlocken Crescent, Suite 130 Broomfield, C.O. 80021, U.S.A.

Richard, C.F. (1992). Energy in farm production. (Elsevier publishing: Netherlands).

Rubinstein, R.Y. (1981). Simulation and the Monte Carlo method. New York: John Wiley and Sons, Inc. Sciences. Taylor \& Francis Group, Boca Raton, FL. 378p.

Shrestha, D.S. (1998). Energy use efficiency indicator for agriculture. http://www.usaskca/agriculture/caedac/PDF/mcrae.PDF; Accessed, March 2012.

Singh, A. K.; Goyal, V.; Mishra, A. K. and Parihar, S. S.( 2013). Validation of CropSyst simulation model for direct seeded ricewheat cropping system. CURRENT SCIENCE, VOL. 104, NO. 10, 25.

Tester, J. ( 2005). Sustainable energy : choosing among options. Cambridge,Mass.: MIT Press.

Verma, S. (1987). Energy in production agriculture and food processing. Prpceeding of the National Conference held at the Punjab Agricultural University ,Ludhana. October 30-31.

Villada, J. and Olaya, Y. (2013), A simulation approach for analysis of short-term security of natural gas supply in Colombia, Energy Policy, 53, 11-26.

Winston, W.L. (1991). Operations Research Applications and Algorithms (2nd Ed.).

Woolfson, M. M. and Pert, G.J. (1999). An Introduction to Computer Simulation, Oxford New York, 1999.

Yilmaz, I.; Akcaoz, H. and Ozkan, B. (2005).An analysis of energy use and input costs for cotton production in Turkey. Renew. Ene. $30: 145-155$. 


\section{الملخص العربى}

\section{نموذج محاكاة مونت كارلو لاستهلاك الطاقة في انظمة إنتاج القمح تحث الظروف المصرية

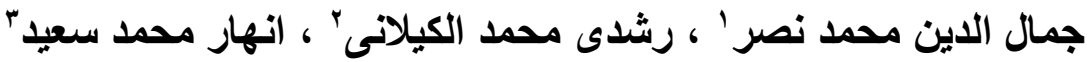

يهدف هذا البحث الى استخدام أسلوب محاكاة مونت كارلو لدراسة انتشار عدم التيقين في نظم إنتاج القمح تحت الظروف المصرية .ولتحقيق هذا الهدف تم استخدام تسعة عشر نظام لانتاج القمح بدءا من عمليات اعداد وتجهيز مرقد البذرة حتى عمليات الحصاد و الدر اس والتذرية. وتم

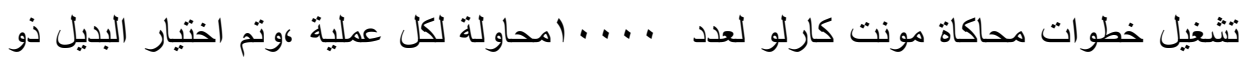
التردد العالي في كل عملية وكذلك تم حساب الطاقة المستهكة في كل نظام ـ وتم التوصل من خلال النتائج المتحصل عليها الى ظهور نظي فلى جديد بالإضافة إلى التسعة عشر نظام محل الدر اسة وكان إجمالي الطاقة المستهكة في هذا النظام 10205.09ميجا جول/ فدان. يتكون هذا النظام من المحراث الحفار ( وجه واحد ) + المشط القرصي + القصابية الحديدية + البتانة + آلة التسطير المعلقة +الري السطحي + نثر السماد يدويا + الرشاشة الظهرية المحمولة لمكافحة الحشائش و الحشرات+ الكومباين. وكان النظام الثالث عشر أكثر الأنظمة استهلاكا للطاقة من ضمن النظم التسعة عشر محل الدراسة وكان مقدار الاستهلاك 14650.8ميجا جول/ فدان في

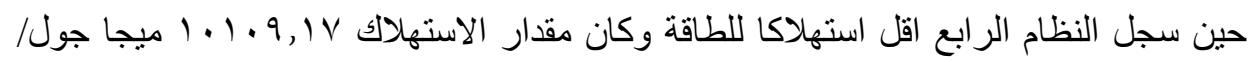
فدان. يتكون هذا النظام من المحراث المطرحي + المشط القرصي + الزحافة الخشبية + البتانة + آلة التسطير المعلقة + الري السطحي + نثر السماد بدويا + الرشاشة الظهرية المحمولة لمكافحة الحشائش و الحشر ات+ محشة ذاتية الحركة+ المقطورة+ الة الدر اس الثابتة. يمكن الاستنتاج من نتائج هذه الدراسة أن نموذج محاكاة مونت كارلو قادر على تحديد النظام

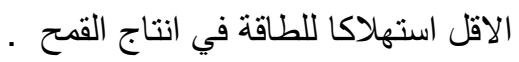

' استاذ الهندسة الزراعية ـ كلية الزراعة - جامعة القاهرة.

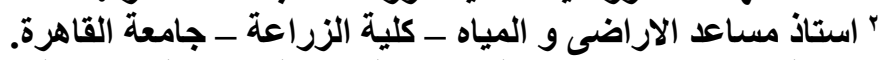

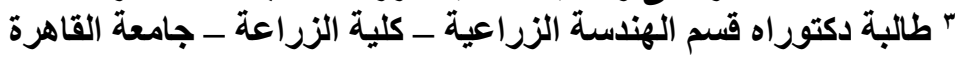

